\title{
ÁRBOLES VIEJOS COMO INDICADORES DE BIODIVERSIDAD DE VERTEBRADOS FORESTALES AMENAZADOS DE LA PROVINCIA DE SALAMANCA (ESPAÑA)
}

\section{Ancient trees as indicators of threatened forest vertebrate biodiversity of Salamanca (Spain)}

\author{
M. Núñez ${ }^{1 *}$, T. Tarazona ${ }^{2}$, F. Silla ${ }^{1}$ y L. Delgado ${ }^{3}$ \\ 'Departamento de Biología Animal y Ecología, Facultad de Biología, Universidad de Salamanca, Campus Unamuno, 37007 , Salamanca, Spain. \\ e-mail: marina.nunezgerman@gmail.com \\ ${ }^{2}$ Servicio Territorial de Medio Ambiente de Salamanca, Sección de Espacios Naturales y Especies Protegidas, C/Príncipe de Vergara 53-71, 37003, Salamanca, Spain. \\ ${ }^{3}$ Departamento de Botánica y Fisiología Vegetal, Facultad de Biología, Universidad de Salamanca, Campus Unamuno, 37007 , Salamanca, Spain. \\ *Autor corresponsal
}

Recibido: 09-12-2015. Aceptado: 11-04-2016. Fecha de publicación on-line: 07/06/2016

Citation / Cómo citar este artículo: M. Núñez, T. Tarazona, F. Silla y L. Delgado (2016). Árboles viejos como indicadores de biodiversidad de vertebrados forestales amenazados de la provincia de Salamanca (España). Pirineos, 171 , e020. doi: http://dx.doi.org/10.3989/Pirineos.2016.171004

RESUMEN: Se ha comparado la abundancia de árboles viejos en la provincia de Salamanca (España) con la distribución de las especies de vertebrados forestales amenazados presentes, observándose que existe una correlación significativa entre ambos parámetros para las siguientes especies estudiadas: águila imperial (Aquila adalberti), buitre negro (Aegypus monachus), lince ibérico (Lynx pardinus), milano real (Milvus milvus) y un grupo de especies de quirópteros forestales (Miniopterus schreibersii, Myotis bechsteinii, Myotis emarginatus, Myotis mystacinus, Myotis myotis, Nyctalus lasiopterus, Nyctalus noctula, Rhinolophus euryale, Rhinolophus ferrumequinum y Rhinolophus mehelyi). Se ha demostrado que existe un incremento de la biodiversidad de vertebrados forestales amenazados paralelo al aumento de la densidad de árboles viejos en los municipios de la provincia. Por ello, se puede deducir que la densidad de árboles viejos es un buen parámetro indicador del estado de conservación del ecosistema forestal y clave para el mantenimiento de dichas especies amenazadas.

PALABRAS CLAVE: Árboles viejos; región mediterránea; vertebrados forestales amenazados; indicador de biodiversidad.

\begin{abstract}
Ancient trees abundance has been compared with distribution of threatened forest vertebrate species at the province of Salamanca (Spain). A significant correlation between both parameters has been observed for the following considered species: imperial eagle (Aquila adalberti), black vulture (Aegypus monachus), iberian lynx (Lynx pardinus), red kite (Milvus milvus) and a group of forest bats (Miniopterus schreibersii, Myotis bechsteinii, Myotis emarginatus, Myotis mystacinus, Myotis myotis, Nyctalus lasiopterus, Nyctalus noctula, Rhinolophus euryale, Rhinolophus ferrumequinum y Rhinolophus mehelyi). It has been proved that there is an increase of threatened forest vertebrates biodiversity along with increasing ancient trees density at the municipalities of the province. Therefore, we can deduce that ancient trees density is a good indicator parameter of the conservation status of the forest ecosystem and it is essential for the maintenance of these endangered species.
\end{abstract}

KEY WORDS: Ancient trees; Mediterranean region; threatened forest vertebrates, biodiversity indicator. 


\section{Introducción}

La provincia de Salamanca cuenta con una superficie forestal de 533.384 hectáreas, donde el hábitat de las dehesas está muy bien representado, suponiendo 320.808 hectáreas (Prieto et al., 1999; Villanueva, 2005). Además, gran parte de estos bosques adehesados son muy antiguos, por lo que existe una gran riqueza de árboles viejos, posiblemente centenarios (De Luis, 1991; Llorente, 1995; Tarazona, 2006). Esta situación permite que mucha de la fauna de vertebrados mediterráneos sea abundante en este territorio (Costa et al., 1990).

En el presente estudio se ha relacionado la densidad de árboles viejos centenarios con la distribución y presencia de vertebrados forestales amenazados en la provincia de Salamanca. Se ha pretendido averiguar si los árboles viejos constituyen una de las variables de las que depende la presencia de dicha fauna amenazada. Podría considerarse, en tal caso, a los árboles viejos como indicador de la calidad del hábitat forestal y de la biodiversidad (Lindenmayer et al., 2000).

\section{Material y Métodos}

\subsection{Los árboles viejos de Salamanca}

El área de estudio coincide con los límites de la provincia de Salamanca y abarca una superficie de $12.349 \mathrm{~km}^{2}$. La base de datos provincial de árboles viejos consta de 1.547 registros. Se han excluido los registros de los árboles que no se encontraban en un hábitat forestal, así como los árboles de especies ornamentales, analizándose finalmente 1.491 de los árboles viejos del inventario, pertenecientes a 35 especies: Acer monspessulanum, Alnus glutinosa, Arbutus unedo, Betula alba, Buxus sempervirens, Castanea sativa, Celtis australis, Crataegus monogyna, Fagus sylvatica, Fraxinus angustifolia, Ilex aquifolium, Juglans regia, Juniperus communis, Juniperus oxycedrus, Morus nigra, Pinus pinaster, Pinus pinea, Pinus sylvestris, Pyrus communis, Pyrus pyraster, Populus nigra, Prunus avium, Quercus ilex subsp. ballota, Quercus faginea subsp. faginea, Quercus faginea subsp. lusitanica, Quercus pyrenaica, Quercus robur, Quercus suber, Salix alba, Sorbus aria, Taxus baccata, Tila cordata, Tila platyphyllos, Ulmus minor y Ulmus nigra. Las especies de árboles con mayor número de registros se indican en la Figura 1. En esta base de datos se inventariaron las encinas (Quercus ilex subsp. ballota) de más de $1 \mathrm{~m}$ de diámetro estándar (DAP) y, en el caso del resto de las especies, los individuos de más de 0,5 m de DAP (Ceballos et al., 1971; Asociación Española de Parques y Jardines Públicos, 1999; Palacios, 2004; Villanueva, 2005; López et al., 2009; Herranz, 2011). Menos del 10\% de estos árboles están protegidos por el Decreto 63/2003 (Junta de Castilla y León, 2003, BOCyL No 100). Se ha calculado la densidad de árboles viejos $\left(\mathrm{n}^{\circ}\right.$ de pies $\left./ \mathrm{km}^{2}\right)$ en cada municipio y se ha realizado una cartografía mediante ESRI®Arc Map ${ }^{\mathrm{TM}} 10.0$.

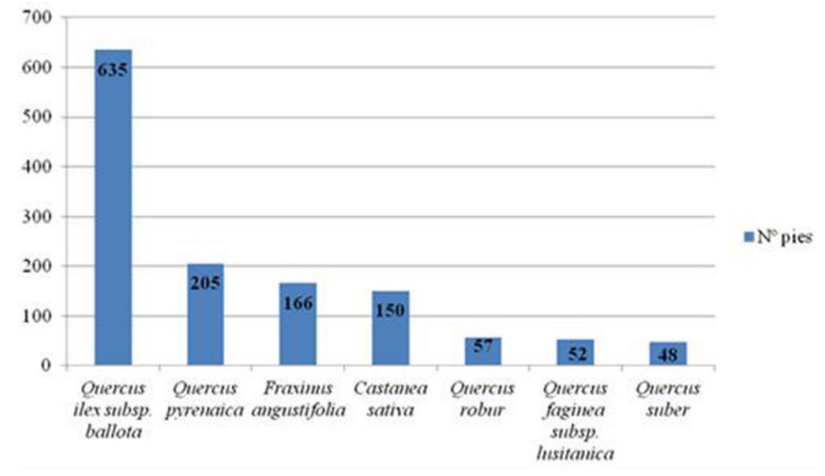

Figura 1: Número de árboles de las especies con más registros del estudio.

Figure 1: Number of trees of the most recorded species at the studio.

\subsection{Vertebrados forestales amenazados de Salamanca}

La selección de las especies de vertebrados a estudiar se ha realizado en base al Catálogo Español de Especies Amenazadas del Real Decreto 139/2011 (Ministerio de Medio Ambiente y Medio Rural y Marino, 2011, BOE No 46), que define sus diferentes categorías de amenaza. De este modo, se han considerado todas las especies de vertebrados amenazados presentes en la provincia de Salamanca que estuvieran ligadas al hábitat forestal, excepto el desmán ibérico (Galemys pyrenaicus), por no disponer de datos suficientes para realizar el análisis. En total, se ha trabajado con diecisiete especies, tres catalogadas en peligro de extinción (EN) y el resto como vulnerables (VU). Las especies concretas de vertebrados forestales amenazados que se han incluido en el estudio son las siguientes: águila imperial (Aquila adalberti, EN), águila-azor perdicera (Hieraaetus fasciatus, VU), alimoche común (Neophron percnopterus, VU), cigüeña negra (Ciconia nigra, VU), buitre negro (Aegypus monachus, VU), milano real (Milvus milvus, EN), lince ibérico (Lynx pardinus, EN) y diez especies de quirópteros catalogadas como vulnerables (Miniopterus schreibersii, Myotis bechsteinii, Myotis emarginatus, Myotis mystacinus, Myotis myotis, Nyctalus lasiopterus, Nyctalus noctula, Rhinolophus euryale, Rhinolophus ferrumequinum y Rhinolophus mehelyi).

Los datos disponibles sobre la distribución de estas especies están basados en nidificaciones para Aegypus monachus y Milvus milvus y en citas de avistamientos para el resto de las especies. En el caso de Milvus milvus, se consideraron las referencias de nidificaciones del censo de 2008, en el cual Salamanca se situaba como la provincia española con mayor número de milano reproductor. (Peris, 1991; Peris et al., 1999; Proyecto LIFE/ NATURALEZA B4-3200-96-511, 2001; Martí \& del Moral, 2004; Proyecto LIFE/NATURALEZA B4-3200-94740, 2004; Proyecto LIFE/NATURALEZA 95/265, 2005; Sanz-Zuasti \& Velasco, 2005; Velasco et al., 2005; Blan- 
co, 2007; Palomo et al., 2007; Hernández, 2010). Con la información disponible sobre la distribución de estas especies en la provincia de Salamanca, se ha elaborado una cartografía básica de presencia/ausencia por términos municipales de cada una de ellas, utilizando ESRI®Arc Map $^{\mathrm{TM}}$ 10.0. Debido al bajo número de datos disponibles a nivel de especie de los quirópteros amenazados, se ha optado por tratarlos como grupo.

\subsection{Análisis estadístico}

Se ha comparado la distribución de las especies de vertebrados forestales amenazados con la distribución de los árboles viejos de la provincia para inferir posibles correlaciones estadísticas. Los análisis correspondientes a Hieraaetus fasciatus y Neophron percnopterus han actuado como control negativo ya que, aunque sean vertebrados amenazados en la provincia, son especies que no requieren el hábitat forestal para su reproducción.

Se han realizado Modelos Lineales Generalizados (GLMs) para determinar los patrones de presencia/ausencia, en los municipios, de las diferentes especies animales estudiadas de acuerdo a una variable explicativa: la densidad de árboles viejos. Se ha usado el programa estadístico R.3.1.1 ${ }^{\circledR}$ y las variables dependientes se han ajustado a un modelo binomial (Quinn \& Keough, 2002). El análisis pretende establecer una relación, predecible y aplicable, entre la presencia de cada especie y la densidad de árboles viejos. La ecuación del modelo, donde $\pi(\mathrm{x})$ indica la probabilidad de encontrar la especie de vertebrado forestal amenazado, se describe de la siguiente forma:

$$
\ln \left(\frac{\pi(x)}{1-\pi(x)}\right)=\beta_{0}+\beta_{1} \text { (variable explicativa) }
$$

En segundo lugar, con los vertebrados forestales amenazados en los que se demostró una vinculación con la densidad de árboles viejos en el primer análisis, se ha realizado un Modelo Linear Generalizado con R.3.1.1 ${ }^{\circledR}$, ajustando la variable dependiente a un modelo binomial negativo. Con ello, se ha pretendido estudiar cómo influye la densidad de árboles viejos en el número de especies de vertebrados forestales amenazados que aparecen en los municipios, para determinar un posible patrón de riqueza de especies de vertebrados forestales amenazados en cada municipio como respuesta a la misma variable explicativa del primer análisis (densidad de árboles viejos).

\section{Resultados}

En la Figura 2 se muestra la densidad de pies de árboles viejos en cada municipio de la provincia de Salamanca.

El cartografiado de la densidad de pies de árboles viejos muestra dos zonas con valores más altos, en el norte y

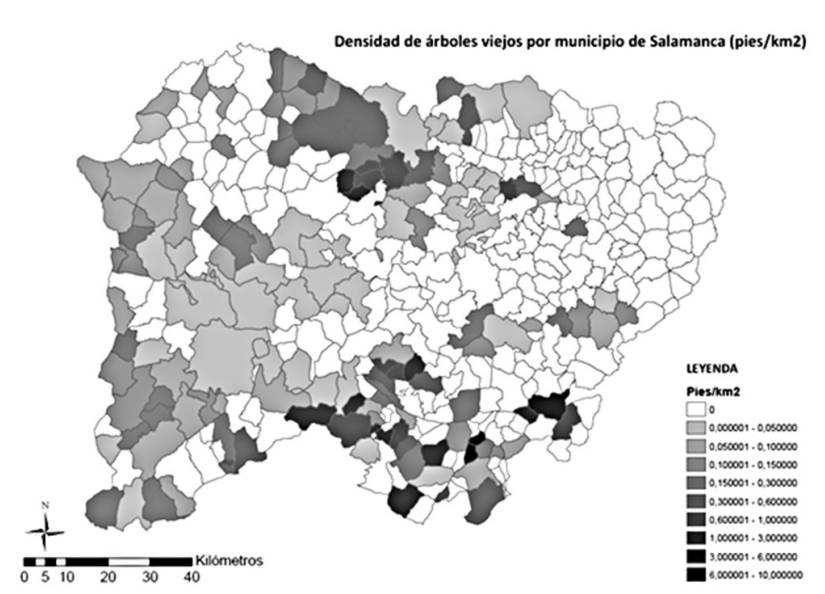

Figura 2: Densidad de árboles viejos por municipio de Salamanca, representados en gama de grises en función del número de pies de árboles viejos por $\mathrm{km}^{2}$.

Figure 2: Ancient tree density by municipality of Salamanca, represented in grey scale according to number of feet of ancient trees per $\mathrm{km}^{2}$.

en el sur, unidas por una franja que recorre el oeste de la provincia, coincidiendo la ausencia de árboles viejos con la zona de cultivos de la mitad este.

\subsection{Modelos Lineales Generalizados (GLMs).}

Los resultados se han considerado estadísticamente significativos si " $p$ " $<0,05$. Según este requisito, los análisis estadísticos realizados para estudiar la presencia/ausencia de los vertebrados forestales amenazados en respuesta a la variable explicativa "densidad de árboles viejos" resultaron significativos para: Aquila adalberti, Aegypus monachus (nidificante), Lynx pardinus, Milvus milvus (nidificante) y el conjunto de los quirópteros amenazados. Por el contrario, los resultados no fueron significativos para: Hieraaetus fasciatus y Neophron percnopterus. En el caso de Ciconia nigra, se obtuvo un valor de " $p$ " cercano a la significación.

Las Figuras 3-10 representan la probabilidad de encontrar la especie estudiada en función de la densidad de pies de árboles viejos. Por otro lado, se obtuvieron los coeficientes necesarios para elaborar la ecuación del modelo.

El análisis estadístico correspondiente al estudio de la riqueza de los vertebrados forestales amenazados se ha realizado tomando en cuenta las especies en las que se demostró una relación significativa con los árboles viejos, es decir, Aquila adalberti, Aegypus monachus, Lynx pardinus, Milvus milvus y el conjunto de los quirópteros amenazados, incluyéndose también Ciconia nigra, ya que roza la significación. El GLM resultó significativo con un valor de $p=0.00635$. La curva de riqueza de especies se muestra en la Figura 11 e indica una relación directa y positiva entre la densidad de árboles viejos de los municipios y el número de estos vertebrados forestales presentes en dichos municipios. 


\section{- SIGNIFICATIVOS}

$$
\begin{aligned}
& \begin{array}{l}
\text { Águila } \\
\text { imperial } \\
\text { (Aquila }
\end{array} \quad \ln \left(\frac{\pi(x)}{1-\pi(x)}\right)=-0,582+0,7564 \text { (Densidad de pies de árboles viejos } / \mathrm{km}^{2} \text { ) } \\
& \text { adalberti) } \\
& \mathrm{p}=\mathbf{0 . 0 2 3 8}
\end{aligned}
$$

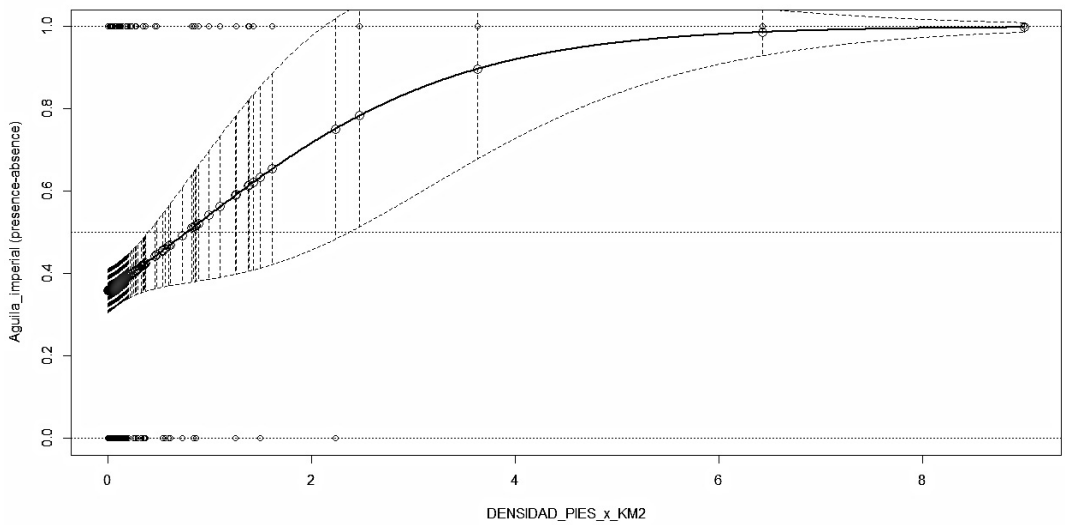

Buitre negro

(Aegypius monachus)

$\ln \left(\frac{\pi(x)}{1-\pi(x)}\right)=-2,2898+0,3644$ (Densidad de árboles viejos $\left./ \mathrm{km}^{2}\right)$ $\mathbf{p}=\mathbf{0 . 0 4 1 2}$

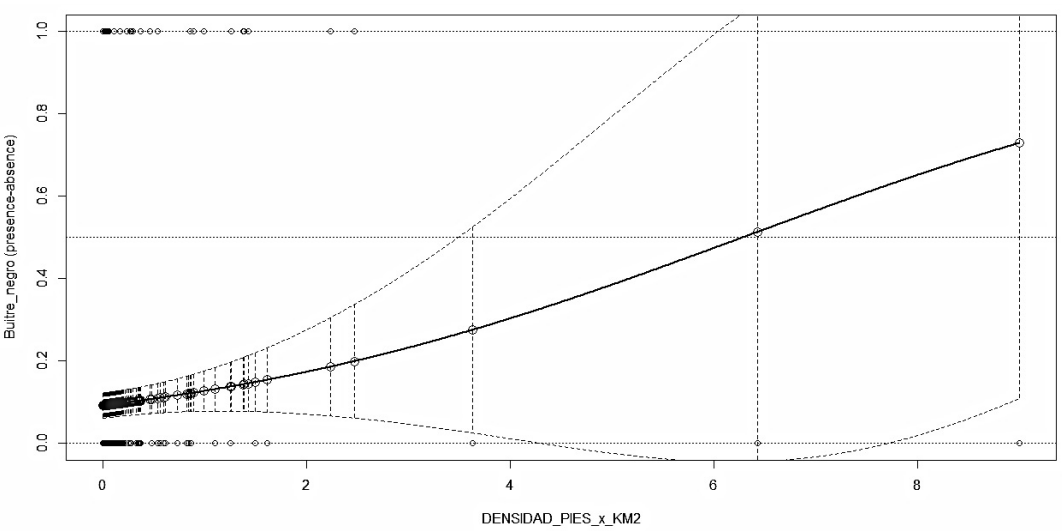

$$
\begin{array}{cc}
\begin{array}{c}
\text { Lince } \\
\text { ibérico } \\
(\text { Lynx }
\end{array} & \left.\ln \left(\frac{\pi(x)}{1-\pi(x)}\right)=-0,9186+1,8178 \text { (Densidad de árboles viejos } / \mathrm{km}^{2}\right) \\
\text { pardinus }) & \mathbf{p}=\mathbf{0 . 0 0 0 1 8 4}
\end{array}
$$

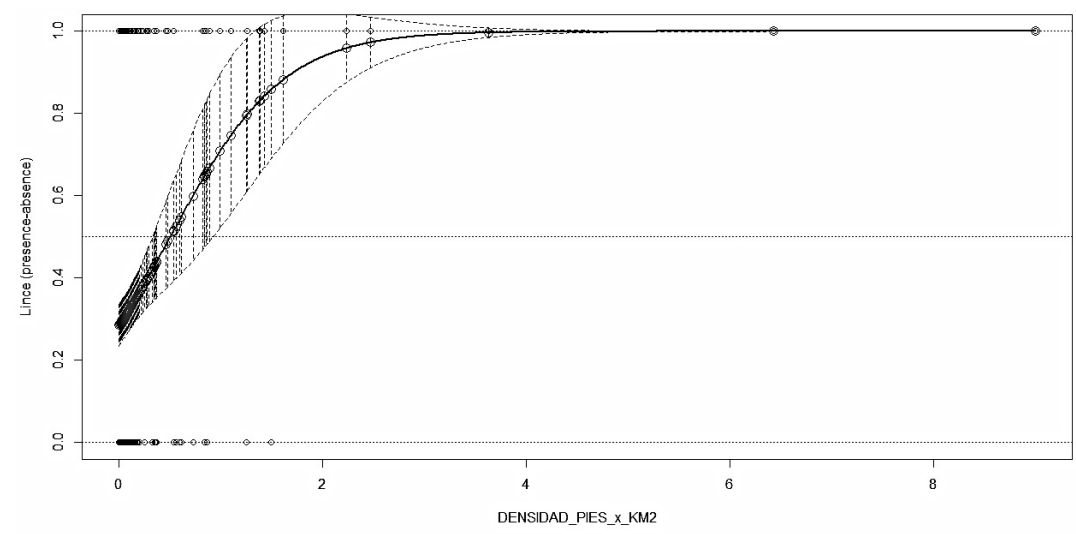

Figura 3: Probabilidad de encontrar águila imperial (Aquila adalberti) en función de la densidad de árboles "centenarios" por municipio $[\pi(\mathrm{x})]$ y ecuación del modelo.

Figure 3: Probability of finding imperial eagle (Aquila adalberti) according to ancient tree density by municipality $[\pi(x)]$ and model equation.

Figura 4: Probabilidad de encontrar buitre negro (Aegypius monachus) en función de la densidad de árboles "centenarios" por municipio $[\pi(\mathrm{x})]$ y ecuación del modelo.

Figure 4: Probability of finding black vulture (Aegypius monachus) according to ancient tree density by municipality $[\pi(x)]$ and model equation.

Figura 5: Probabilidad de encontrar lince ibérico (Lynx pardinus) en función de la densidad de árboles "centenarios" por municipio $[\pi(\mathrm{x})] \mathrm{y}$ ecuación del modelo.

Figure 5: Probability of finding iberian lynx (Lynx pardinus) according to ancient tree density by municipality $[\pi(x)]$ and model equation. 


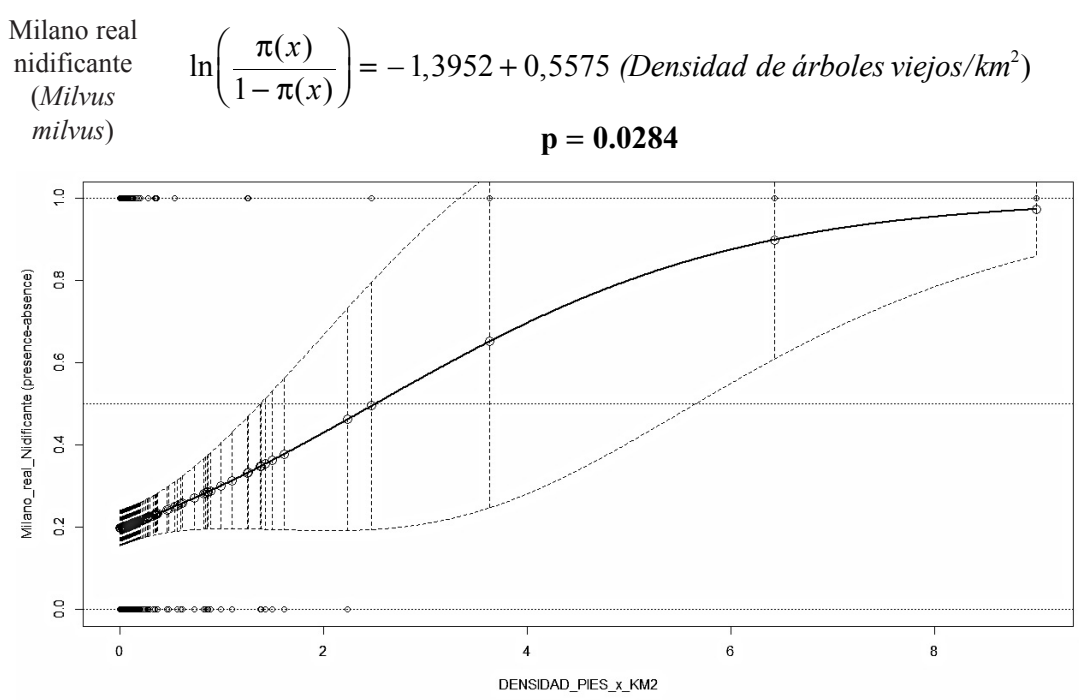

Quirópteros

forestales

$\ln \left(\frac{\pi(x)}{1-\pi(x)}\right)=-0,1758+1,0813$ (Densidad de árboles viejos $\left./ \mathrm{km}^{2}\right)$

$\mathbf{p}=\mathbf{0 . 0 1 2 1}$

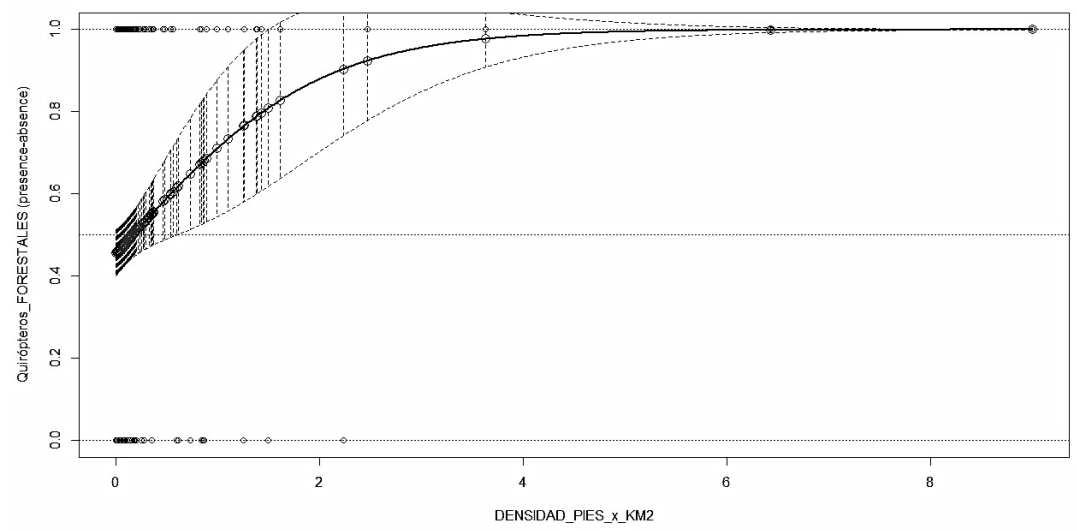

\section{- CERCANO A LA SIGNIFICACIÓN}

Cigüeña negra (Ciconia nigra)

$\ln \left(\frac{\pi(x)}{1-\pi(x)}\right)=-0,0443+0,4454$ (Densidad de árboles viejos $\left./ \mathrm{km}^{2}\right)$

$\mathbf{p}=\mathbf{0 . 1 2 3}$

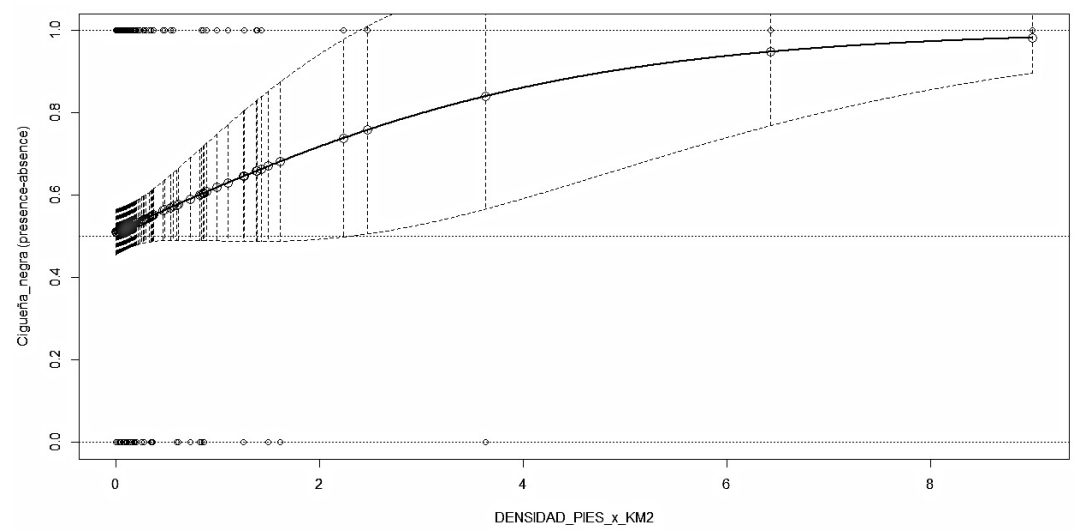

Figura 6: Probabilidad de encontrar milano real nidificante (Milvus milvus) en función de la densidad de árboles "centenarios" por municipio $[\pi(\mathrm{x})] \mathrm{y}$ ecuación del modelo.

Figure 6: Probability of finding nesting red kite (Milvus milvus) according to ancient tree density by municipality $[\pi(x)]$ and model equation.

Figura 7: Probabilidad de encontrar quirópteros forestales amenazados en función de la densidad de árboles "centenarios" por municipio $[\pi(\mathrm{x})]$ y ecuación del modelo.

Figure 7: Probability of finding threatened forest bats according to ancient tree density by municipality $[\pi(x)]$ and model equation.
Figura 8: Probabilidad de encontrar cigüeña negra (Ciconia nigra) en función de la densidad de árboles "centenarios" por municipio $[\pi(\mathrm{x})]$ y ecuación del modelo.

Figure 8: Probability of finding black stork (Ciconia nigra) according to ancient tree density by municipality $[\pi(x)]$ and model equation. 


\section{- NO SIGNIFICATIVOS}

Águila-azor

perdicera

(Hieraaetus

fasciatus)

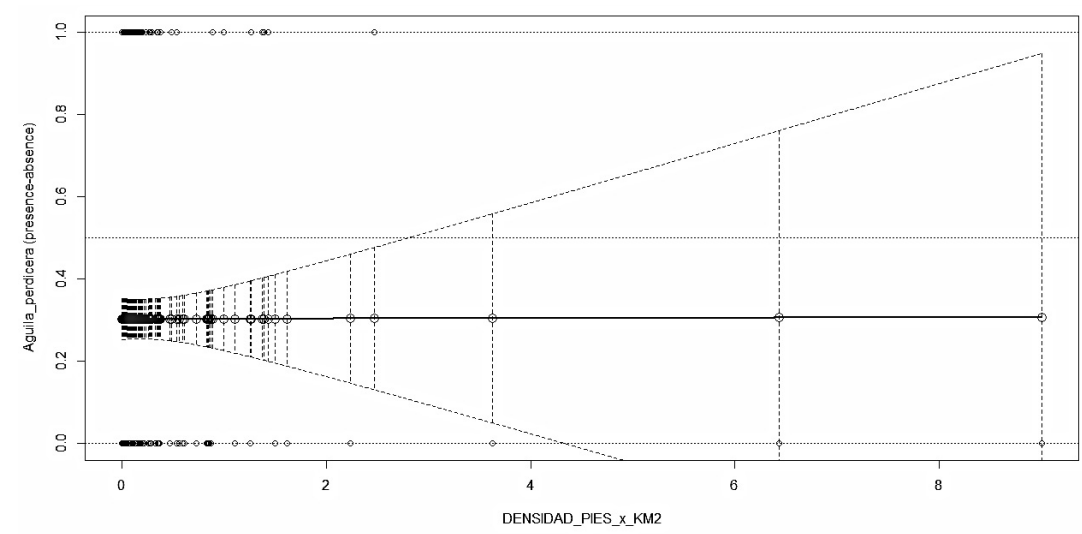

Alimoche

común

(Neophron

Percnopterus)
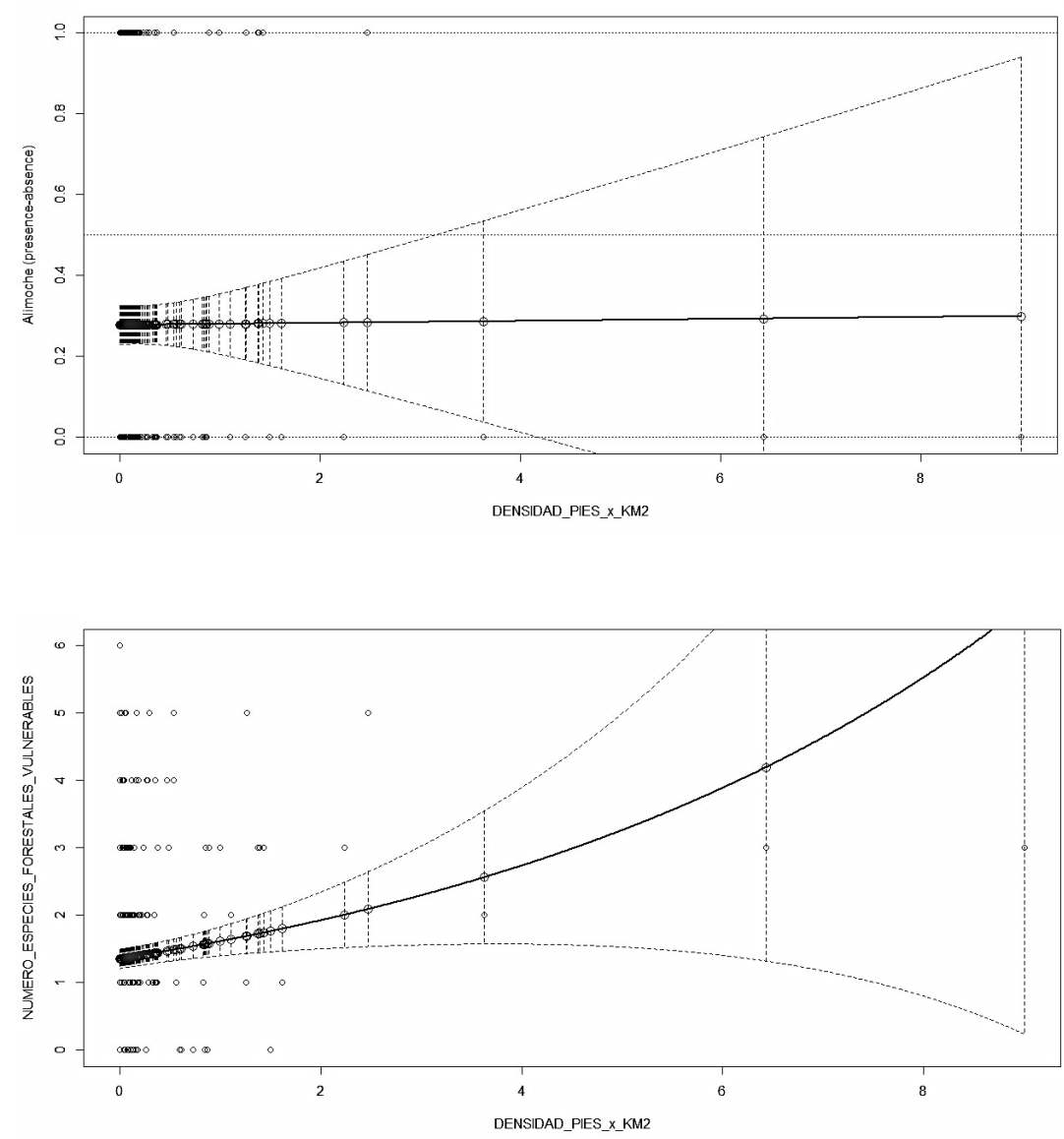

Figura 9: Probabilidad de encontrar águila-azor perdicera (Hieraaetus fasciatus) en función de la densidad de árboles "centenarios" por municipio $[\pi(\mathrm{x})]$.

Figure 9: Probability of finding Bonelli's eagle (Hieraaetus fasciatus) according to ancient tree density by municipality $[\pi(x)]$

Figura 10: Probabilidad de encontrar alimoche común (Neophron percnopterus) en función de la densidad de árboles "centenarios" por municipio $[\pi(\mathrm{x})]$.

Figure 10: Probability of finding egyptian vulture (Neophron percnopterus) according to ancient tree density by municipality $[\pi(x)]$

Figura 11: Número de especies de vertebrados forestales amenazados asociados a los árboles "centenarios" en función de la densidad de árboles "centenarios" por $\mathrm{km}^{2}$.

Figure 11: Number of species of threatened forest vertebrates associated to ancient trees according to density of ancient trees per $\mathrm{km}^{2}$. 


\section{Discusión}

Los resultados del análisis estadístico fueron significativos y probaron una relación positiva entre la presencia de la especie y la densidad de árboles viejos para Aquila adalberti, Aegypus monachus (nidificante), Lynx pardinus, Milvus milvus (nidificante) y el grupo de los quirópteros amenazados (Miniopterus schreibersii, Myotis bechsteinii, Myotis emarginatus, Myotis mystacinus, Myotis myotis, Nyctalus lasiopterus, Nyctalus noctula, Rhinolophus euryale, Rhinolophus ferrumequinum y Rhinolophus mehelyi). Es decir, la densidad de árboles viejos favorece el asentamiento de estas especies, de forma que es más probable encontrarlas donde mayor es la densidad de árboles viejos. Por otro lado, se vislumbra una tendencia clara hacia la significación en el caso de Ciconia nigra.

Para Aegypus monachus y Milvus milvus se ha trabajado estrictamente con datos referentes a nidificaciones, lo que indica que la relación de estas especies con los árboles viejos es directa y está íntimamente relacionada con sus conductas reproductivas. Eligen hábitats forestales para nidificar en grandes árboles y usan un espectro de ambientes más amplio para conseguir alimento, como matorrales abiertos o dehesas. Su distribución y abundancia actuales y futuras están condicionadas por la disponibilidad de árboles grandes adecuados para instalar el nido (Morán et al., 2007; Iberis, 2008).

Para el caso de Aquila adalberti, Ciconia nigra y el grupo de quirópteros amenazados, se ha trabajado con citas de avistamientos (Junta de Castilla y León, 1995, BOCyL No 92/1995; Consejería de Medio Ambiente, 2002, BOCyL No 214; Junta de Castilla y León, 2003, BOCyL $\mathrm{N}^{\mathrm{o}} 195$ ), por lo que la relación con los árboles viejos es menos directa y puede indicar que requieren un hábitat con una cobertura arbórea de cierta importancia para que la reproducción se lleve a cabo. Para estas especies, la densidad de árboles viejos indica la presencia de un bosque maduro, con baja intensidad de actividad humana y buena cobertura arbórea (González et al., 1990; Hernández, 2010; Cano, 2012). Además, los resultados obtenidos para Ciconia nigra, en base a las áreas propuestas para su plan de recuperación y a datos de censos recientes (Junta de Castilla y León, 1995, BOCyL No 92/1995; Consejería de Medio Ambiente, 2002, BOCyL No 214), rozan la significación y encajan con el comportamiento adaptativo observado en esta especie hacia la nidificación rupícola, que puede precisamente derivar de la falta de un hábitat forestal de calidad. Es decir, Ciconia nigra depende en menor medida de la presencia de árboles viejos porque ha conseguido adaptarse a nidificar en las rocas como segunda opción (Oikos, 2010; Cano, 2012). Respecto al conjunto de los quirópteros, Schwendtner et al. (2005) indican que requieren la presencia de un gran número de huecos, donde refugiarse y encontrar alimento, que pueden ser encontrados en los árboles viejos del bosque.

La zona de presencia de Lynx pardinus con la que se ha trabajado es el área de importancia para esta especie en Salamanca, propuesta por el Servicio Territorial de Medio
Ambiente de Salamanca (2001) y elaborada en función de más 235 citas recopiladas desde 1994 hasta 2005. Dada la naturaleza de los datos empleados para este caso, los resultados pueden interpretarse como un requerimiento de hábitat de esta especie y pueden usarse como información para las gestiones de reintroducción y adecuación de corredores. Según Palomares et al. (2000), los individuos jóvenes, durante la fase de dispersión, requieren masas de pinos y, en el momento del asentamiento, eligen zonas de matorral mediterráneo pero protegidas por zonas boscosas. Palomares (2001) describe una diferencia clara entre el hábitat de dispersión y el de asentamiento, según la cual los árboles altos son característicos de la fase de dispersión para dotar de protección y los árboles pequeños son característicos de la fase de asentamiento. Según Ferreras et al. (2004), la hembra elige zonas protegidas para alumbrar a las crías, como oquedades de troncos viejos de alcornoques o nidos viejos en árboles, hecho que se ha comprobado en Doñana. En Salamanca, hasta los años 90 hay datos de muertes de linces adultos y crías, por lo que se puede establecer la zona como área de dispersión y de núcleos reproductores ocasionales (Rodríguez \& Delibes, 1990; Grande del Brío, 1993; Tarazona \& Pablos, 2006).

Los resultados estadísticamente negativos para Hieraaetus fasciatus y Neophron percnopterus eran esperables, pues se trata de especies no ligadas a un ambiente forestal, que nidifican en roca. De ese modo, se comprueba que han actuado en el análisis realizado como controles negativos. Las áreas de cría suelen situarse en los exteriores de las sierras, asentando sus nidos en cavidades de barrancos de amplio rango de alturas (Ceballos \& Donázar, 1989; Real, 2004; Junta de Castilla y León, 2006, BOCyL $\mathrm{N}^{\circ} 230$ ). Por tanto, los árboles viejos no son buenos indicadores de su presencia, ya que no los necesitan para criar.

Está bien documentado que los bosques maduros deben de tener prioridad en la protección forestal por su papel como fuente de diversidad (Lindenmayer et al., 2000; Schwendtner et al., 2005). La presencia de árboles viejos o "centenarios" es un indicador de la existencia de bosques maduros. Por tanto, el conocimiento de la selección de hábitat de especies amenazadas es muy importante para mejorar las acciones dirigidas a su conservación (Lindenmayer et al., 2000). Los programas de reintroducción de especies también deberían tener en cuenta los análisis de los patrones globales de selección del hábitat de las especies consideradas (Calzada et al., 2010). Tras haber tenido en cuenta la biología reproductiva de las especies amenazadas estudiadas mediante este trabajo, observamos el vínculo directo de su conducta reproductiva con la presencia de árboles viejos.

La cartografía de árboles viejos y de las especies animales forestales amenazadas sujeto de este estudio también ha permitido aclarar si la densidad de pies de árboles viejos "centenarios" condiciona la riqueza de estas especies por municipio. Se ha comprobado que las áreas de distribución de los vertebrados forestales amenazados estudiados coinciden con las zonas con mayor número de árboles viejos en la provincia de Salamanca. Además, los municipios más 
ricos en especímenes viejos de árboles tienen mejores características para que se establezca un mayor número de las especies de vertebrados estudiadas. La densidad de los árboles viejos se confirma como un buen indicador de la calidad del hábitat forestal y, por tanto, de la biodiversidad y riqueza en especies de dicho hábitat.

\section{Conclusión}

En este artículo se ha demostrado que la densidad de árboles viejos es un buen indicador de la riqueza y biodiversidad de vertebrados forestales amenazados y, por tanto, de la calidad de los hábitats forestales de Salamanca. Los árboles viejos constituyen un elemento directamente relacionado con la conducta reproductiva de los vertebrados estudiados, demostrando su importante vinculación. Cabe recalcar la necesidad de proteger los árboles viejos, como clave para poder mantener y conservar los hábitats forestales y, con ello, asegurar la presencia y supervivencia de, al menos, los siguientes vertebrados forestales amenazados de Salamanca: águila imperial (Aquila adalberti), buitre negro (Aegypus monachus), lince ibérico (Lynx pardinus), milano real (Milvus milvus) y el conjunto de los quirópteros amenazados estudiados (Miniopterus schreibersii, Myotis bechsteinii, Myotis emarginatus, Myotis mystacinus, Myotis myotis, Nyctalus lasiopterus, Nyctalus noctula, Rhinolophus euryale, Rhinolophus ferrumequinum y Rhinolophus mehelyi).

\section{Agradecimientos}

Queremos agradecer a los Agentes Medioambientales $\mathrm{y}$ a todas aquellas personas que han proporcionado información sobre los árboles y las especies animales de este estudio.

\section{Referencias}

Asociación Española de Parques y Jardines Públicos, 1999. Método para la valoración de árboles y arbustos ornamentales. Norma Granada.

Blanco, M. (Ed.), 2007. Anuario Ornitológico de la provincia de Salamanca, 2004-2006. SEO-Salamanca.

Cano, L.S., 2012. Efecto de la ubicación del nido y de la gestión del territorio sobre la productividad de la cigüeña negra $(\mathrm{Ci}$ conia nigra) en España. En: Cano, L.S.: Memoria para optar al grado de Doctor (2012): Biología y conservación de la cigüeña negra (Ciconia nigra) en la Península Ibérica. Universidad Complutense de Madrid.

Calzada, J., Mora, M., Giles, R. \& Márquez, C., 2010. Lince ibérico: aspectos jurídicos para la conservación de la especie. SECEM, Málaga.

Ceballos, L., de Córdoba, F. \& Ruiz, J., 1971. Árboles y arbustos de la España peninsular. Escuela Técnica Superior de Ingenieros de Montes. Sección de publicaciones.

Ceballos, O. \& Donázar, J.A., 1989. Factors influencing the breeding density and nest-site selection by the Egyptian Vulture (Neophron percnopterus). Journal für Ornitbologie. 130(3): 353-359. http://dx.doi.org/10.1007/bf01644748
Consejería de Medio Ambiente de la Junta de Castilla y León, 2002. Orden de 10 de julio de 2002, por la que se declaran veintisiete nuevas áreas críticas para la Conservación de la Cigüeña negra (Ciconia nigra) en su Zona de Importancia para la conservación de la especie en las provincias de Ávila, Salamanca y Segovia. BOCyL No 214.

Costa, M., García-Antón, J., Morla, C. \& Sainz, H., 1990. La evolución de los bosques en la Península Ibérica: una interpretación basada en datos paleobiogeográficos. Ecología, Fuera de Serie. $N^{\circ} 1$ : 31-58. I.C.O.N.A., Madrid.

De Luis, E., 1991. Bioclima. En: Gómez, J.M. (Coord.): El libro de las dehesas salmantinas. Junta de Castilla y León, Consejería de Medio Ambiente y Ordenación del Territorio.

Ferreras, P., Delibes, M., Palomares, F., Fedriani, J.M., Calzada, J. \& Revilla, E., 2004. Proximate and ultimate causes of dispersal in the Iberian Lynx (Lynx pardinus). Behavioural Ecology. 15 (1): 31-40. http://dx.doi.org/10.1093/beheco/ $\arg 097$

González, L.M., Bustamante, J. \& Hiraldo, F., 1990. Factors influencing the present distribution of the Spanish Imperial Eagle (Aquila adalberti). Biological Conservation. 51: 311319. http://dx.doi.org/10.1016/0006-3207(90)90116-7

Grande del Brío, R., 1993. El lince ibérico. Amarú Ediciones. Salamanca.

Hernández, L., 2010. Murciélagos de la provincia de Salamanca, conservación y estudio de los murciélagos salmantinos. SECEMU.

Herranz, C., 2011. Las guías del Duero: Árboles únicos de Castilla y León. El Mundo.

Iberis S.A., 2008. Censo de la población de Milano real de Castilla y León. Dirección General del Medio Natural de la Consejería de Medio Ambiente de la Junta de Castilla y León. Servicio Territorial de Salamanca.

Junta de Castilla y León, 1995. Decreto 83/1995, de 11 de mayo, por el que se aprueba el Plan de Recuperación de la Cigüeña negra y se dictan medidas complementarias para su protección en la Comunidad de Castilla y León, establece las Zonas de Importancia para la Conservación de la Cigüeña negra. BOCyL No 92/1995.

Junta de Castilla y León, 2003. Decreto 63/2003, de 22 de mayo, por el que se regula el Catálogo de Especímenes Vegetales de Singular Relevancia de Castilla y León y se establece su régimen de protección. BOCyL $\mathrm{N}^{\mathrm{o}} 100$.

Junta de Castilla y León, 2003. Decreto 114/2003, de 2 de octubre, por el que se aprueba el Plan de Recuperación del Águila Imperial Ibérica y se dictan medidas para su protección en la Comunidad de Castilla y León. BOCyL No 195

Junta de Castilla y León, 2006. Decreto 83/2006, de 23 de noviembre, por el que se aprueba el Plan de Conservación del Águila Perdicera en Castilla y León. BOCyL N 230.

Lindenmayer, D.B., Margules, C.R. \& Botkin, D.B., 2000. Indicators of biodiversity for ecologically sustainable forest management. Conservation Biology, 14(4): 941-950. http:// dx.doi.org/10.1046/j.1523-1739.2000.98533.x.

Llorente, J. M., 1995. La dehesa salmantina. Medio Ambiente en Castilla y León, 4: 25-34.

López, C., Espinosa, J. \& Bengoa, J., 2009. Mapa de vegetación de Castilla y León. Síntesis 1:400.000. Junta de Castilla y León. Consejería de Medio Ambiente.

Martí, R. \& del Moral, J.C., 2004. Atlas de las aves reproductoras de España. Parques Nacionales.

Ministerio de Medio Ambiente y Medio Rural y Marino, 2011. Real Decreto 139/2011, de 4 de Febrero, para el desarrollo del Listado de Especies Silvestres en Régimen de Protección Especial y del Catálogo Español de Especies Amenazadas. BOE No 46.

Morán, R., Sánchez, J.M., Costillo, E \& Villegas, A., 2007. El buitre negro en masas de monte mediterráneo. En: 
Moreno-Opo, R. y Guil, F. (Eds.): Manual de gestión del hábitat y de las poblaciones de buitre negro en España. Ministerio de Medio Ambiente, pp. 179-196, Madrid.

Oikos S.L., 2010. Conservación y Gestión de la Biodiversidad y el Territorio. Informe parcial extraído de informe final "Análisis del fracaso de la productividad de la población de cigüeña negra y revisión de la zonificación de las áreas de importancia para la conservación de la especie en Castilla y León".

Palacios, C.J., 2004. Guía de los árboles singulares de España. Editorial Blume, S. A., 128 pp., Barcelona -Puerto del Rosario.

Palomares, F., 2001. Vegetation structure and prey abundance requirements of the Iberian Lynxs: implications for the design of reserves and corridors. Journal of Applied Ecology, 38(1): 9-18. http://dx.doi.org/10.1046/j.1365-2664.2001.00565.x.

Palomares, F., Delibes, M., Ferreras, P., Fedriani, J.M., Calzada, J. \& Revilla, E., 2000. Iberian Lynx in a fragmented landscape: predispersal, dispersal, and postdispersal habitats. Conservation Biology, 14 (3): 809-818. http://dx.doi. org/10.1046/j.1523-1739.2000.98539.x.

Palomo, L.J., Gisbert, J. \& Blanco, J.C., 2007. Atlas y Libro Rojo de los Mamíferos Terrestres de España. Dirección General para la Biodiversidad - SECEM - SECEMU, 588 pp., Madrid.

Peris, S., 1991. Los mamíferos, las aves y los peces. En: Gómez, J. M. (Coord.): El libro de las dehesas salmantinas. Junta de Castilla y León, Consejería de Medio Ambiente y Ordenación del Territorio.

Peris, S.J., Reyes, E. \& Hernández, L., 1999. Atlas de mamíferos silvestres de la provincia de Salamanca. Diputación de Salamanca, Naturaleza y Medio Ambiente.

Prieto, A., Puerto, A., Para, J.L. \& Martín, R., 1999. Especies de flora y fauna de la dehesa salmantina: abundancia relativa, intervención humana y regulación. Revista de Estudios, 42: Salamanca.
Proyecto LIFE NATURALEZA B4-3200/94/740, 2004. Lince: Área de Rodríguez y Delibes. Junta de Castilla y León.

Proyecto LIFE NATURALEZA 95/265, 2005. Lince: Área de Sierra de Gata. Junta de Castilla y León.

Proyecto LIFE/NATURALEZA B4-3200-96-511, 2001. Inventario de refugios y atlas de distribución de los quirópteros de Castilla y León. Junta de Castilla y León.

Quinn, G.P. \& Keough, M.J., 2002. Experimental design and data analysis for biologists. Cambridge University Press. http://dx.doi.org/10.1017/cbo9780511806384.

Real, J., 2004. Águila-azor perdicera (Hieraaetus fasciatus). En: Madroño, A.; González, C. y Atienza, J.C. (Eds.): Libro Rojo de las Aves de España. Dirección General para la Biodiversidad-SEO/Birdlife. Madrid.

Rodríguez A. \& Delibes M., 1990. El Lince Ibérico en España. Distribución y problemas de conservación en España. ICONA, Ministerio de Agricultura, Pesca y Ganadería.

Sanz-Zuasti, J. \& Velasco, T., 2005. Guía de Aves de Castilla y León. Nueva edición revisada y ampliada. Náyade Editorial. Medina del Campo (Valladolid).

Schwendtner, O., Recalde, I., \& Alcalde, J.T., 2005. Importancia de los árboles senescentes y la madera muerta en la gestión de los bosques naturales. Congresos Forestales.

Tarazona, T., 2006. Las dehesas de Salamanca y la Red Natura 2000. L'Atazavara, 15: 69-72.

Tarazona T. \& Pablos C., 2006. La conservación del lince ibérico en Salamanca, 1995-2005. Conferencia impartida el 24 de junio 2006 dentro del Título propio de la Universidad de Salamanca: Experto universitario en Gestión y Conservación de Flora y Fauna y Espacios Protegidos.

Velasco, J.C., Lizana, M., Román, J., Delibes, M. \& Fernández J., 2005. Guía de los Peces, Anfibios, Reptiles y Mamíferos de Castilla y León. Náyade Editorial. Medina del Campo (Valladolid).

Villanueva, J.A., 2005. Tercer inventario forestal nacional (1997-2007). Ministerio de Medio Ambiente, Madrid. 